\title{
Using BIM to mitigate risks associated with health and safety in the construction and maintenance of infrastructure assets
}

\author{
D. Ruikar \\ School of Civil Engineering University of Birmingham, UK
}

\begin{abstract}
Building Information Modelling (BIM) is a descriptive term for the technologically advanced, collaborative and information-centric processes used to drive design, construction and operation of the built environment. BIM is essentially value creating collaboration through the entire life-cycle of an asset (building or infrastructure), underpinned by the creation, collation and exchange of shared 3D models and intelligent, structured data attached to them. BIM is also about a shift in culture that will affect the way the built environment and infrastructure works and how it is delivered. Historically the health and safety record of the construction industry in the UK is relatively poor as compared to the manufacturing industries. BIM and the digital environment it operates within now allow us to use design and construction data in a more intelligent way. It allows data generated by the design process to be re-purposed and contribute to improving efficiencies in other areas of a project. This evolutionary step in design is not only creating exciting opportunities for the designers themselves but it is also creating opportunity for every stakeholder in any given project. From designers, engineers, contractors through to H\&S managers, BIM is accelerating a cultural change. The paper describes the idea behind a research project at the University of Birmingham that uses BIM as a platform to elucidate construction, operation and maintenance health hazards and using the principles of prevention devise mechanisms for their control.
\end{abstract}

Keywords: health and safety, BIM, asset management, CDM regulations, heat map, risk ranking, health hazards, risk ranking. 


\section{Introduction}

Building Information Modelling (BIM) is a descriptive term for the technologically advanced, collaborative and information-centric processes used to drive design, construction and operation of the built environment. BIM is essentially value creating collaboration through the entire life-cycle of an asset (building), underpinned by the creation, collation and exchange of shared 3D models and intelligent, structured data attached to them. BIM is often seen as just $3 \mathrm{D}$ modelling, however it is much more than that. It is also about a shift in culture that will affect the way the built environment and infrastructure works and how it is delivered. BIM is not simply about the technology and tools; that have enabled and led to an evolution in the way design teams design and deliver. As technology advances, BIM models are becoming increasingly sophisticated and data rich which benefits designers, clients and collaborators alike. These advances create opportunities for improving facility management, maintenance and retrofitting of buildings. Wider use will streamline work processes and alter the relationship between different parties in the construction industry, leading to improved coordination. The health and safety $(H \& S)$ function associated with the design and construction of the infrastructure asset is no exception. Historically the H\&S record of the construction industry in the UK is relatively poor as compared to the manufacturing industries (HSE [1]). This was one of the key reasons for the introduction of a Construction, Design and Management (CDM) co-ordinator at the design stage of construction projects (both building and infrastructure). The CDM co-ordinator is tasked with co-ordinating H\&S information especially at the design phase of the project to ensure there is co-operation amongst the design team with respect to $\mathrm{H} \& \mathrm{~S}$ data/information. The introduction of $\mathrm{CDM}$ role has contributed to the "safe by design" principle and a drastic drop in the number of fatalities per year in the UK construction sector (HSE [1]). According to the HSE (Health and Safety Executive) fatalities in UK construction industry have almost halved today as compared to the early 1990s when they used to be over a 100 a year. However in spite of these improvements the construction industry still lags behind the manufacturing sector in terms of its H\&S record. BIM and the digital environment it operates within now allow us to use design and construction data in a more intelligent way. It allows data generated by the design process to be repurposed and contribute to improving efficiencies in other areas of a project. This evolutionary step in design is not only creating exciting opportunities for the designers themselves but it is also creating opportunity for every stakeholder in any given project. From designers, engineers, contractors through to H\&S managers, BIM is accelerating a cultural change. The paper describes the idea behind a newly commenced research project at the University of Birmingham that uses BIM as a platform to elucidate construction, operation and maintenance health hazards and using the principles of prevention devise mechanisms for their control. The research paper investigates and reports on the outline idea of this research project which investigates as to how CDM coordinators and H\&S mangers working within a BIM environment can apply suitable health rating to the infrastructure assets that are being designed and constructed. 


\section{BIM adoption in UK}

\subsection{BIM definition}

The term Building Information Modelling (BIM) has been subject to a number of different definitions proposed by a number of academics. Of these definitions, the one proposed by Eastman et al. [2] is found to be the most comprehensively acknowledged definition. BIM is defined by Eastman et al. [2] as a: '...verb or adjective phrase used to describe tools, processes and technologies that are facilitated by digital machine-readable documentation about a building, its performance, its planning, its construction, and its operation'. BIM therefore may be fundamentally defined as an activity, rather than an object. As summarised by Pikas et al. [3], the output of this activity is the building information model or simply the building model, which represents a form of (visual) information repository. This building information model forms the basis of a digital database, which holds specific information in relation to a particular building and information pertaining to its objects. Information is held in the form of machinereadable parametric objects that are able to be read by BIM software tools, capable of compiling models of buildings in their virtual form.

\subsection{Levels and stages of BIM maturity}

BIM is still yet to be fully realised in terms of its potential for development, and its level of adoption within the AEC industry in the UK. This concept was explored by Bew and Richards [4] in 2008, who proposed a formal BIM maturity model, through which an understanding of the steps required to implement BIM incrementally has been derived (SEC [5]). This framework has been adhered to extensively under best practice guidance, and by the UK government in their framework for the adoption of BIM (SEC [5]; BIMWP [6]). The Bew Richards maturity model, presented in figure 1, divides the adoption of BIM into three distinct maturity levels, evolving from the traditional construction paradigm to the fully integrated adaptation of BIM. Parallel research proposed by Succar [7] exemplifies those levels of the above model, under which an alternate framework is proposed. Whilst Succar [7] refers to the increments as stages, it should be noted that each increment is in strict alignment with the levels of the other framework suggested by Bew and Richards [4]. Succar [7] identifies: '... Integrated Project Delivery (IPD) to denote an approach to or an ultimate goal of implementing BIM, as the fundamental goal encompassing each component of BIM. The three stages proposed by Succar [7] are as follows:

- BIM Stage 1 (Level 1): object-based modelling;

- BIM Stage 2 (Level 2): model-based collaboration, and;

- BIM Stage 3 (Level 3): network-base integration.

Each stage should be implemented gradually and in logical order of increment, due to the weight of their impact in influencing 'transformational' changes. 


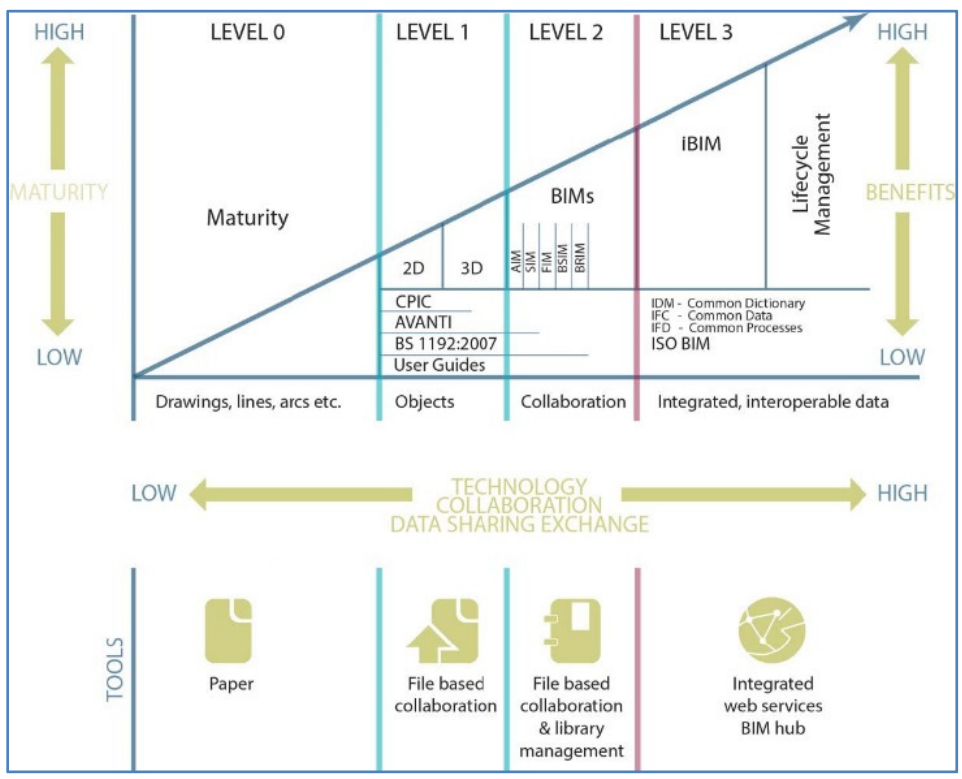

Figure 1: $\quad$ Bew and Richards BIM maturity model [4].

\subsubsection{Pre-BIM}

Pre-BIM denotes the nature of traditional construction practices used prior to the adoption of BIM, from which a number of barriers and inefficiencies may be recognised (Khosrowshahi and Arayici [8]).

\subsubsection{BIM Stage 1: object-based modelling}

Stage 1 represents the initial stage under which BIM is formally adopted in the fundamental transition from 2D documentation to the use of 3D object-based modelling (Succar [7]). This single-disciplinary model forms the basis of coordination between 2D documentation and 3D visualisation, to lead advancements over pre-BIM (Khosrowshahi and Arayici [8]). BIM is still in its infancy at this stage and thus, the single disciplinary model is unable to improve collaboration between the disciplines to influence any significant improvements in communication. It is highlighted by Succar [7] that the key benefits at this stage are derived from the potential to support the undertaking of design and construction processes concurrently, to reduce construction times.

\subsubsection{BIM Stage 2: model-based collaboration}

Stage 2 finds its definition in the progression made from modelling to the interoperability of disciplines, with which communication and collaboration are augmented (Succar [7]). This collaboration is dependent on the sharing of data between disciplines through integrated data communication. This sharing of data is defined by the SEC [5] to encompass the interoperability of the following four components: Platform software(s); Database(s); Specialist design analysis tools, and; Mechanisms for asset data drops. 
The transition from traditional construction practices becomes notable at this stage as the adoption of BIM begins to change existing cultures; redefining roles, disciplines and existing barriers between project phases (Succar [7]). It should also be noted that amendments to contract strategies become a requirement at this stage to accommodate the nature of these changes.

\subsubsection{BIM Stage 3: network-based integration}

Level 3 represents the aspirations of BIM to embody its underlying principles in a transition from collaboration to becoming a fully integrated solution, at which stage the BIM models assume the characteristics of fully interdisciplinary $\mathrm{nD}$ (multi-dimensional) models (SEC [5]). Prior cultures of the traditional construction paradigm are redefined extensively to erode associated barriers to accommodate real-time collaboration between parties. These changes are significant in enabling BIM to be exploited to its most heightened potential, and moreover to enable an increased emphasis on virtual workflows as defined by Khosrowshahi and Arayici [8].

\subsection{BIM adoption in the UK}

Having discussed the three levels of BIM maturity, it would be appropriate to consider these trends in the context of the relevant maturity levels adopted amongst those organisations currently using BIM in the UK, in parallel with the relevant government requirements.

Recent research has implied that the industry has gained significant momentum in its overall adoption of BIM, following the catalytic impact of the announcement of the Construction Strategy by the UK government in 2011 (Dave et al. [9]). Within this report, the Cabinet Office reported that the UK construction industry is characteristically fragmented, as a result of the minimal notion of collaboration amongst individual disciplines in the industry; a sentiment reverberated in research by Arayici et al. [10], by whom it was discussed that the UK construction industry is faced with uncertainties and conflicts with current traditional practices. The Construction Strategy Report (Cabinet Office [11]) formally acknowledged these barriers as the basis under which the report was derived, in alignment with best practice outlined in the Egan Report [12] to advance an increased collaboration in construction. It was recognised that whilst significant benefits may be realised through the adoption of BIM, there is typically widespread incompatibility between other disciplines in its current form. Within the Construction Strategy, it was proposed that: '...the government will require a fully collaborative 3D BIM (with all project and asset information, documentation and data being electronic) by the end of 2016', under which BIM level 2 will become a mandatory requirement in the development of all public sector projects from 2016.

In the lead up to the mandatory adoption of BIM, studies have indicated that there is significant attestation to the widespread adoption of BIM level 1, with further indication of level 2 having been adopted by a number of organisations in advance of the 2016 deadline. These findings are substantiated within the National BIM Report 2014 (NBS [13]), in which it is found that $51 \%$ of the industry have formally adopted BIM up to level 1, with a further $31 \%$ of organisations within 
the UK having achieved level 2 prior to the mandatory deadline. The distribution of maturity levels achieved amongst the industry indicates that more than $7 \%$ of the industry have made significant progress to achieve BIM level 3 on a number of projects that they have undertaken. However, despite these indications toward the early adoption of BIM, it is important to observe that nearly two thirds of the industry are still yet to reach level 2 by 2016, of which $11 \%$ of the organisations are still reliant on Pre-BIM standards and traditional construction practices.

BIM represents a significant change in the flow of information from the traditional paradigm of construction to one which encompasses construction management principles based on BIM. The adoption of BIM leads to an increase in communication amongst those parties involved in a project, made more efficient through the elimination of the traditional and inefficient paper based approach to the exchange of project data and information. Through the facilitation of greater accuracy in the design stage, BIM may be adopted to reduce the necessity for rework, and reduce the impact of waste during the construction process. It is evident that the overall impact of these advantages may be derived to deliver enhanced value, and lead to significant improvements to production over traditional construction practices. However BIM is still very much an emerging technology, and as a consequence there are still a number of barriers to the adoption of BIM within the industry. Fragmentation amongst individual organisations and disciplines stands out as one such notable impediment, typified by an evident necessity for the change in culture required to enable the adoption of BIM. Further barriers have been identified concerned with the initial commitment to the initiative in terms of training and implementation costs, as well as those associated with potential legal issues regarding ownership and design responsibility.

The aspirations of the UK Government to adopt BIM on all public sector projects have been formally recognised, and as of 2016 will form a mandatory requirement. To promote the standardisation of BIM a number of standards have been written for example the requirements for the adoption of maturity level 2 are addressed within PAS 1192-2:2013 specifically.

\section{Health and safety in UK construction}

The construction industry is notorious for its health and safety record in comparison with other industries. For decades, construction has been the most dangerous industry to work in despite the drastic improvements that have been made over the years. The information in the table below shows the drastic decrease over the past decades in the amount of incidents that have occurred on site. This has been as a result of many changes that have been made such as the establishment of CDM, and documents such as the Egan [12] and the Latham [14] Reports (HSE [1]). Although the information in table 1 shows good development in relation to $\mathrm{H} \& \mathrm{~S}$ over the years, there is still a lot of room for improvement. In order to continually improve, the construction industry must keep up with the latest technologies; this is where BIM can potentially play a significant role. By 
Table 1: $\quad H \& S$ comparison (1974-2011) [1].

\begin{tabular}{|c|c|c|}
\hline Summary description & Year & \\
\hline $\begin{array}{l}\text { Workplace injury (latest data adjusted to align with } \\
1974 \text { reporting requirements) }\end{array}$ & 1974 & 2013 \\
\hline Fatal injuries to employees & 651 & 95 \\
\hline Rate of fatal injury per 100000 employees & 2.9 & 0.4 \\
\hline $\begin{array}{l}\text { Reported non-fatal injuries to employees (2001/12 } \\
\text { figure) }\end{array}$ & 336,701 & 77,310 \\
\hline Occupational diseases & 1974 & 2011 \\
\hline $\begin{array}{l}\text { Deaths from pneumoconiosis as underlying cause } \\
\text { (non-asbestosis) }\end{array}$ & 453 & 152 \\
\hline $\begin{array}{l}\text { Deaths from asbestosis without mention of } \\
\text { mesothelioma (Asbestosis register) }\end{array}$ & 74 & 429 \\
\hline Deaths from mesothelioma (Mesothelioma register) & 243 & 2,291 \\
\hline $\begin{array}{l}\text { Rate of self-reported work-related illness (latest data } \\
\text { adjusted to align with } 1990 \text { survey definitions where } \\
\text { possible) }\end{array}$ & 1990 & 2012 \\
\hline Overall rate per 100000 employed & 5,940 & 3,665 \\
\hline $\begin{array}{l}\text { Rate of Musculoskeletal disorders per } 100000 \\
\text { employed }\end{array}$ & 2,750 & 1,500 \\
\hline $\begin{array}{l}\text { Rate of stress and related conditions per } 100000 \\
\text { employed }\end{array}$ & 820 & 1,520 \\
\hline
\end{tabular}

implementing BIM with regulations such as CDM, there can still be significant improvements.

Safety in construction remains a big problem with frequent loss of life, injuries, near misses and collateral damage that can be prevented. Safe construction requires care and planning throughout the project life cycle, from design, through construction planning, through construction execution and extending into operation and maintenance (Zhang et al. [15]). Failure in any of these areas can increase the risk of exposing workers to hazards in the construction environment. The HSE reported that injuries in workers in construction cost society an estimated $£ 0.7$ billion in $2012 / 13$. The graph below shows the number of fatalities has dropped in construction but it is still a serious concern. The construction industry accounts for about $5 \%$ of the workforce in the UK but it still accounts for $31 \%$ of fatal injuries to employees and 10\% of major/specified injuries (HSE [1]). A study of construction project features by Manu et al. [16] identified that different features have an influence on accident occurrence of high or moderate potential. For example, demolition and refurbishment (high) relative to new work (moderate); high level construction and underground construction (high) relative to low level construction (moderate); tight project duration (high) relative to 


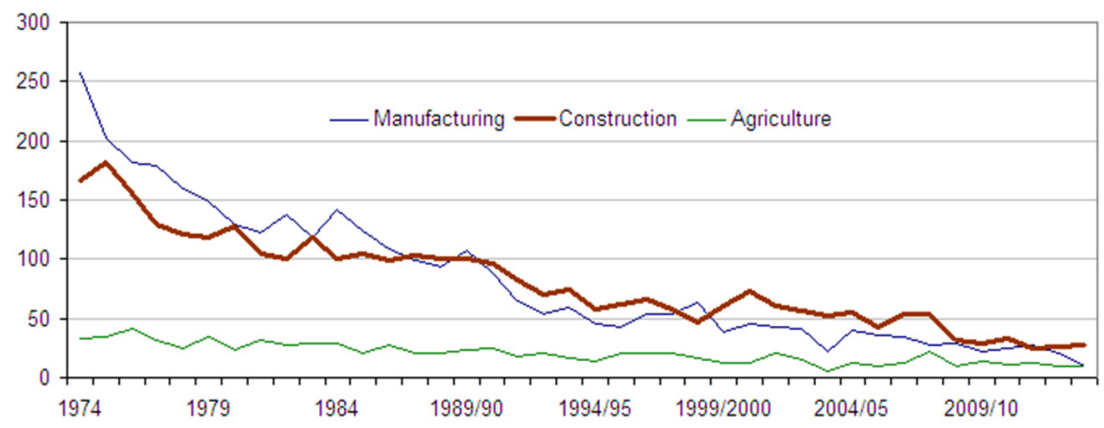

Figure 2: HSE fatality statistics (1974-2013) [1].

adequate project duration (moderate); complex design (high) relative to simple design (moderate).

Incorporating H\&S data within BIM can increase the focus on identifying high risk schemes and raise awareness so that risks and accidents are reduced in the future. Compared to the existing standards, the new regulations have more collaborative approach and the majority of the changes are focused mainly to the management and the coordinating roles, which makes it more applicable to BIM projects and the information management procedures described in PAS 119$2: 2013$ [17]. One of the major changes compared to the 2007 version is that a Principal designer (PD) role is now assigned to coordinate the H\&S during the preconstruction period replacing the one of the CDM Coordinator (CDMC). The changes means more responsibility for coordinating the H\&S during the preconstruction to the designer instead of a third party specialist consultant .The reasons are so that the role should be undertaken by a professional that could be easily integrated into the project team and have the necessary competence and ability to influence the design, which were issues experienced with the CDMC role under the 2007 regulations.

The use of BIM from an H\&S perspective to remove or minimalize risk in the construction process is still being realized within the construction industry. The UK Government although taking a lead on mandating level 2 BIM does not seem to have considered in-depth, BIM as a tool for improving Health and Safety as a major part of its Construction strategy Looking at the UK government construction strategy 2011 there is no mention on the use of BIM and H\&S as being a benefit for the construction industry.

The UK Governments construction strategy 2025 states as one of its visions for construction in 2025 as "an industry that attracts and retains a diverse group of multitalented people, operating under considerably safer and healthier conditions" (HM Cabinet Office [18]). Although there is a mention of a need for improved $\mathrm{H} \& \mathrm{~S}$ there is no mention of BIM being a beneficial tool in achieving this. One step the UK Government made is to commission a working task group called BIM4regs which "aims to incorporate Building Regulations, planning and health and safety requirements into BIM models". It has been commissioned by the 
Government BIM Task Group. The initiative will embed regulatory information within BIM software so architects and engineers can immediately see if designs comply with the rules. The BIM4Regs task group are addressing the fact that checking a building for building regulation compliance can be an automated process and be checked by the designer throughout the production of the BIM model. From an H\&S point of view this automated checking process eliminates human error ensuring that any health and safety issues from noncompliance to regulations are eliminated. After compliance checking for software is incorporated the next step by this task group is to see how judgement based decision making can be incorporated into BIM models. An example of this is given in the NBS document [13] BIM for Construction and H\&S as "the inclusion of data relating to hazardous properties of materials, or with further development, the ability to visualise particular construction scenarios that will inform decisions on subsequent activities or construction methods". Data of hazardous materials can be embedded into the BIM model. For example in a refurbishment project locations containing asbestos could be embedded into the BIM model. Through visualisation site workers can use the model to see which areas or existing elements within the building contain asbestos minimalizing the risk of coming into contact with this hazardous material.

\section{BIM and H\&S}

In construction, traditional safety planning relies on frequent manual observations, is labour intensive and time consuming and thus highly inefficient. Safety knowledge is difficult to transfer by safety regulations alone. Construction site safety often remains the sole responsibility of the contractor. Based on the schedule, construction site changes daily, with new safety issues emerging, some other being removed as the work on site progresses (Zhang et al. [15]). All the hazardous risks possible to occur as a result of these sequences need to be foreseen and safety planning should be completed before the construction work begins. Safe construction requires care and planning throughout the project life cycle, from design through construction planning, through construction execution and extending into operations and maintenance. BIM offers a clear simulation of all these stages, thus enhancing the transfer of knowledge and methods to provide standardization of safe working environment. Furthermore, BIM based modelling and 4D simulation may offer many benefits to safety and logistics applications.

The three areas where BIM can influence H\&S management are: evaluation of site conditions for workers; identification of potential safety hazards; and analysis, planning and validation of safety measures.

Using BIM processes, it is possible to identify potential safety hazards before construction starts onsite. This is one of the crucial factors that help develop improved safety measures. Working with a $3 \mathrm{D}$ model from the beginning of the project, the object visualization is easy. BIM objects should be modelled taking into account its installation/maintenance space requirements and its health and safety too. This can be achieved by adding to their BIM models a CDM BIM object that allows you to place a cone within the model to identify hazards there can be 
different object types to suit the risk as demonstrated in figure 3 (shown as a warning symbol/tag on $2 \mathrm{D}$ outputs). It is then a relatively small step to get designers using an Excel version of a Risk Register, and then use any "Excel Link" tool to assign risks to the CDM "cones" within the BIM model.

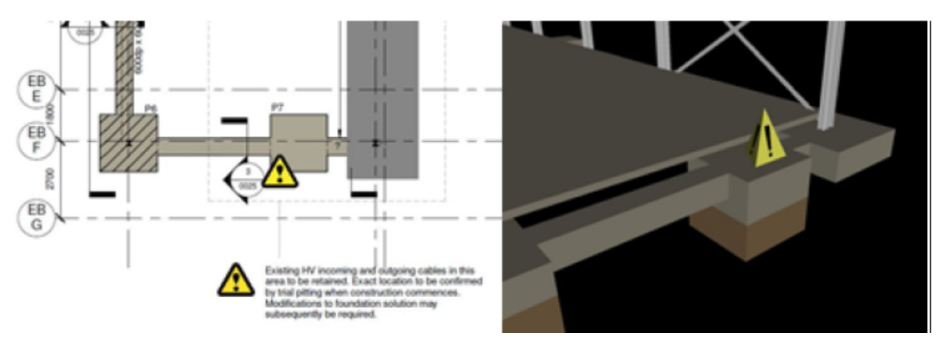

Figure 3: Embedding H\&S objects in BIM.

\section{Conclusion and proposed research project}

Based on the potential use of BIM for H\&S is construction as demonstrated in the paper proposed research will test the approach to using BIM as a platform to elucidate construction, operation and maintenance health hazards and using the principles of prevention devise mechanisms for their control.

As part of this approach it is also the aim to identify a suitable health rating that can be applied to the design to give the contractor an indication of the health exposure to his workers (e.g. high ranking, implies the potential for a high number of exposures to health risk, low ranking implies a lower exposure).

It is proposed that all proposed health \& safety related data that is required to achieve the project aim and objective resides in an Asset Information Management System (AIMS), with the building CAD only storing the associated Asset ID and linking through to AIMS.

To achieve the above, the following steps could be used:

1. Select a structure suitable for 3D modelling (in collaboration with Arup)

2. Structure the model according to the Asset Hierarchy

3. Capture information about the materials used and any know hazards

4. Through construction sequencing, identify and visualise any known health hazards (vibration, noise, carcinogen exposure etc.)

5. Facilitate a health risk identification process that for each asset, evaluates the health hazards and identifies controls

6. Once the health risk identification process is complete undertake a similar assessment for the operation and maintenance of the structure

7. Devise a simple visual rating system that can be applied to the structure

The overall strategy with regards to information management is to keep the CAD data light in terms of attribution, but utilise the AIMS which holds the definitive set of information about building assets (refer to figure 4). To further extend this, a spatial view of the health risks is required as a "heat map" within a $3 \mathrm{D}$ Viewer. To achieve this, the following steps are required: 
1. Perform a gap analysis of existing 3D data (from the BIM model) to AIMS

2. Create a relationship between the individual assets (at entity level if feasible) and the BIM features (on the Asset ID)

3. Develop a heat map based on health risks and their categorisation for display in 3D Viewer

The conceptual architecture of the proposed system can be seen in Figure 4 below.

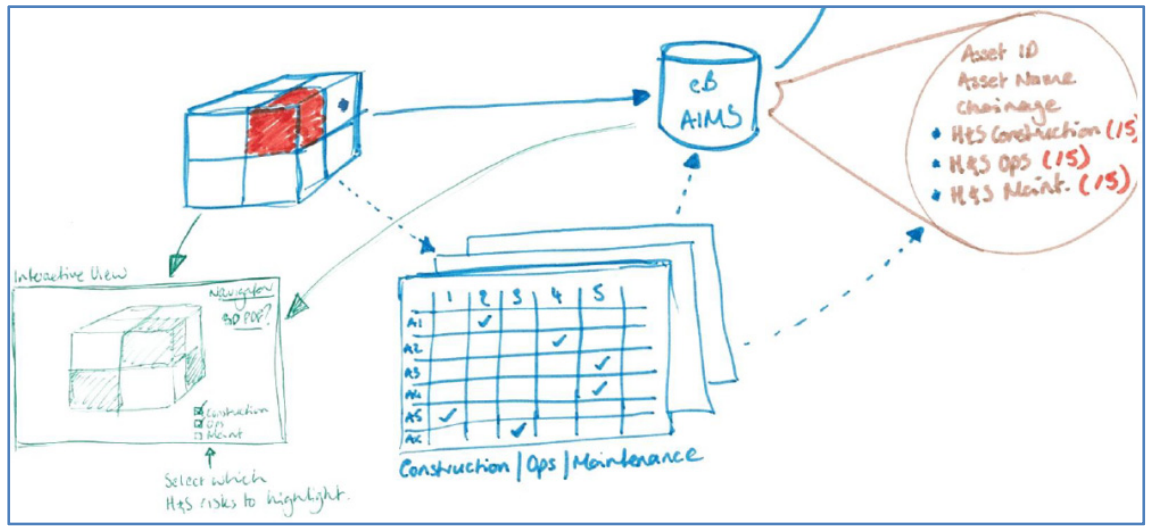

Figure 4: Conceptual architecture and information flow.

Such an approach will give the designers and contractor on-site, an indication of the health risk exposure to their workers (e.g. high ranking, implies the potential for a high number of exposures to health risk, low ranking implies a lower exposure). After all safety during construction and operation is of the concern and responsibilities of all project stakeholders. No matter the project's size and complexity, it should be planned, designed and executed in such way that does not impose a risk to others.

\section{References}

[1] HSE, Health and safety in construction in Great Britain, October 2014. [Online]. Available from: http://www.hse.gov.uk/statistics/construction /construction.pdf 2014.

[2] Eastman, C., Teicholz, P., Sacks, R. and Liston, K. BIM Handbook A Guide to Building Information Modelling for Owners, Managers, Designers, Engineers, and Contractors. New Jersey: John Wiley \& Sons, 2008.

[3] Pikas, E., Koskela, L. Sapountzis, S., Dave, B and Owen, R. Overview of building information modelling in healthcare projects. In: Global health infrastructure challenges for the next decade, Delivering Innovation, demonstrating the benefits. Manchester, 26-28 September 2011.

[4] Bew, M. and Richards. M. Bew Richards Maturity Model, 2008. 
[5] SEC. First Steps to BIM Competence A Guide for Specialist Contractors. London: Special Engineering Contractors' (SEC) Group, 2013.

[6] BIMWP. A report for the Government Construction Client Group: Building Information Modelling (BIM) Working Strategy Paper. London: Constructing Excellence, UKGC and Construction Industry Council. 2011

[7] Succar, B. Building information modelling framework: A research and delivery foundation for industry stakeholders. In: Automation in Construction, 18: (3): 357-375, 2009.

[8] Khosrowshahi, F. and Arayici, Y. Roadmap for implementation of BIM in the UK construction industry. In: Engineering, Construction and Architectural Management. 19: (6): 610-635, 2012.

[9] Dave, B., Boddy, S. and Koskela, L. Challenges and Opportunities in Implementing Lean and BIM on and Infrastructure Project. Proceedings of the 21st Annual Conference of the International Group for Lean Construction. Fortaleza, 29 July-2 August 2013.

[10] Arayici, Y., Coates, P., Koskela, L., Kagioglou, M., Usher, C. and O’Reilly, $\mathrm{K}$. Technology adoption in the BIM implementation for lean architectural practice. Automation in Construction, 2011: (20): 189-195, 2011.

[11] Cabinet Office. Industrial Strategy: government and industry in partnership. Construction 2025. Available at https://www.gov.uk/ government/uploads/system/uploads/attachment_data/file/210099/bis1395 5construction2025industrialstrategy 2011.

[12] Egan, J. Rethinking Construction: Report of the Construction Task Force. London: HMSO, 1998.

[13] NBS. NBS National BIM Report 2014. Newcastle upon Tyne: NBS, 2014.

[14] Latham, M. Constructing the Team: Final Report of the Government/Industry Review of Procurement and Contractual Arrangements in the UK Construction Industry. London: HMSO, 1994.

[15] Zhang, S., Teizer, J., Lee, J., Eastman, C.M., Venugopal, M. Building information modelling (BIM) and Safety: Automatic Safety Checking of Construction Models and Schedules. Automation in Construction, 29, 183195, 2013.

[16] Manu, P., Ankrah, N., Proverbs, D., Suresh, S. The health and safety impact of construction project features. Engineering, Construction and Architectural Management, 21 (1), 65-93. ISSN 0969-9988, 2014.

[17] BSI, PAS 1192-2:2013 Specification for information management for the capital/delivery phase of construction projects using building information modelling. London: British Standards Institution, 2013.

[18] Cabinet Office. Government Construction Strategy - May 2011. London: Cabinet Office, 2011. 